\title{
Curiosity and Interaction: making people curious through interactive systems
}

\author{
Rob Tieben ${ }^{1,2}$ \\ r.tieben@tue.nI
}

\author{
Tilde Bekker $^{1}$ \\ m.m.bekker@tue.nl
}

\author{
Ben Schouten ${ }^{1,2}$ \\ ben.schouten@fontys.nl
}

\author{
1: Eindhoven University of Technology \\ Department of Industrial Design \\ P.O. Box 513, Eindhoven, the Netherlands \\ 2: Fontys University of Applied Sciences \\ School of ICT \\ Rachelsmolen 1, 5612 MA, Eindhoven, the Netherlands
}

\begin{abstract}
We explore the concepts of curiosity and interaction: how can we elicit curiosity in public spaces through interactive systems? We have developed a model consisting of five curiosity-evoking principles. In an iterative design research approach, we have explored the design implementations of these principles. Each principle has been evaluated in a school context, giving us insights about its use for changing behaviour through curiosity. The results of this exploration can be used to inform the design of persuasive and playful interactive systems.
\end{abstract}

interaction; curiosity; design research; persuasion; exploration; interactive systems; playful interaction; motivation

\section{INTRODUCTION}

Imagine that you leave the subway, on your way to the exit of the station. When you reach the escalator, you see that the staircase resembles one big piano. Curious, you climb the first step, and hear a musical note. Climbing onward, you play the piano with every step you take.

TheFunTheory's Piano Stairs (2009), as described in the above scenario, create a novel situation in which people are triggered to take the stairs, instead of the escalator. The big piano is clearly out of place in the subway station, makes people curious and invites them to explore. This curiosity is a strong motivator for behaviour, and novelty is just one of the evokers of curiosity. This paper presents our explorative design-research process on eliciting curiosity in public spaces, through an interactive system.

We are interested in how we can use interactive systems to change behaviour in a playful manner. In previous work we have examined how to design for social and physical play of younger children (Bekker et al., 2010) and for social and physical interaction of older adults (Romero et al, 2010) using a variety of motivational approaches. In this paper we describe the development of a model for design using curiosity principles. In our current project, we focus on teenagers in secondary and vocational schools: can we somehow get the attention of those youngsters, and let them act in a different way, while they walk through the corridor of their school? Can we invite them to briefly interact with a system, in a 'walk-through-and-use' situation?

Curiosity is a strong motivator for behaviour, especially for this young target group; after all, curiosity often decreases with age (e.g. Harter, 1981). Through an iterative design-research process, we have identified, implemented and evaluated a series of principles for eliciting curiosity in the context of a school, through an interactive system.

We first give examples of curiosity in design, and an overview of attempts to define curiosity in depth (section 2). Following that, we present our designresearch approach (section 3 ) and the designs, implementations and evaluations that we conducted (section 4). Section 5 presents 
additional influence factors that we identified, followed by a discussion and conclusions (section 6 and 7).

\section{RELATED WORK}

Curiosity, the strong intrinsic desire we living beings have to know or learn something, has been studied since Aristotle wrote his Metaphysics. It is commonly used to draw attention to stories, products and services. Advertisements, giftwrappings, gossip magazines: they all deploy curiosity in order to 'reach' the user. Cialdini (2001) and Vorst (2007, in dutch) both give a large number of real-world examples.

In the design-research literature, we mostly see surprise and ambiguity being studied, both pivotal elements of curiosity. Two inspiring examples are Zheng et al.'s (2007) work on surprise, and Gaver's (2003) series of ambiguous design explorations. Korhonen et al. (2009) have recently introduced their Playful Experiences framework, in which exploration and discovery play an import role. Last but not least, curiosity is a well-known design element in the world of game design: mystery, sensory stimuli and the advance in levels and storyline are examples of the use of curiosity in games (Garris et al., 2001; Yee, 2007).

However, what we miss are design recommendations for evoking curiosity in encounters with interactive systems. How can we make people who encounter an interactive system curious, and how can we elicit explorative behaviour from them?

\subsection{Curiosity in depth}

Curiosity is one of the driving factors of human behaviour. Berlyne (1960, 1967, 1971) defined two dimensions of curiosity (see Fig. 1): on one axis sensory curiosity, such as novel sensations and stimuli, on the other cognitive curiosity, the desire for knowledge. The other dimension ranged from diversive curiosity - actively seeking varied sources of novelty and challenge - to specific curiosity actively seeking depth in one's knowledge and experience with a particular stimulus or activity (Berlyne, 1960; Kashdan et al., 2004).

Berlyne also states that curiosity is induced by novelty, complexity, uncertainty and conflict. Loewenstein (1994) clarified that curiosity reflects a human tendency to make sense of the world, and that we are curious about things that are unexpected or that we cannot explain. Theories describing the curiosity-drive, such as the information-gap theory (Loewenstein, 1994) and the incongruency theory (Rauterberg, 1995) describe the 'gap in our knowledge' that makes us curious: this gap should not be too big, nor too

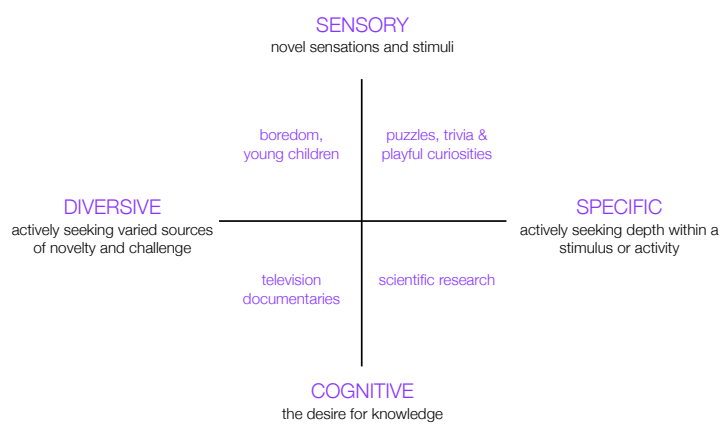

Figure 1: four types of curiosity

small, but exactly 'triggering' enough. Garris et al. (2002) recently showed that curiosity is evoked by incongruity of information, complexity, novelty, surprise, violation of expectations, incompatibility between ideas, inability to predict the future, and information that is incomplete or inconsistent.

Vorst (2007) presents a literature study from the ancient (pre)history to now, summarising what curiosity is, and showing what evokes curiosity; he mainly adds partial exposure (to information and/or stimuli) to the list of evokers, with a striptease as best-known example.

Summarised, we can define five main principles for evoking curiosity: novelty, partial exposure, complexity, uncertainty, and conflict (see figure 2).

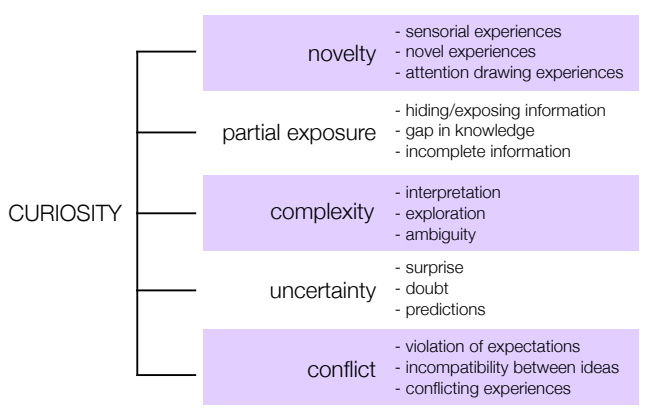

Figure 2: five main principles for evoking curiosity, and some examples

Inspired by Berlyne (1960), Loewenstein (1994) and Kashdan et al. (2004), we visualised the link between the principles, and their role in the explorative process, in Figure 3. 


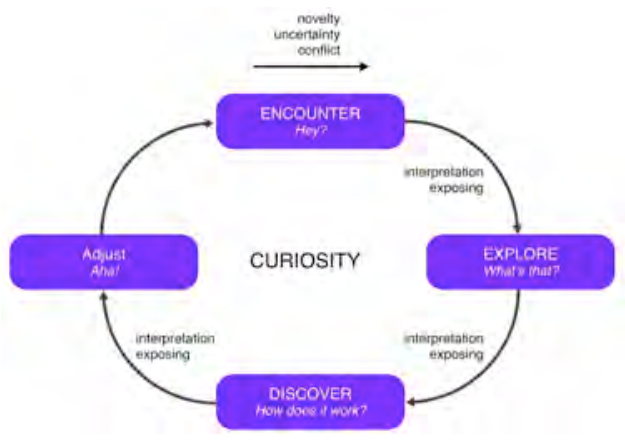

Figure 3: the curiosity process - encountering, exploring, discovering and adjusting. Initiated by novelty, uncertainty and conflict. Driven by interpretation and exposing.

\section{DESIGN-RESEARCH APPROACH}

We used a design-research approach to explore, develop and evaluate different ways to design for curiosity. This was done in an iterative, qualitative and explorative way: through iterations of design and evaluation.

First, various principles for evoking curiosity were identified in literature and real-world examples, as described in the related work. Through iterations of design explorations, we studied the principles in more detail; gaining more understanding of how we could use those principles in interactive systems. Evaluations showed us what sort of explorative behaviour we could elicit with the principles.

In order to facilitate this iterative process, we developed a platform: an interactive prototype in which various explorations could quickly be implemented and evaluated.

We decided to use sound as medium to elicit curiosity: the way in which students walked through a corridor, and the actions they performed, would trigger various sound samples from prototypes on the wall. We developed six prototypes, called speakers, which contained a webcam and a loudspeaker. Interaction was provided using Max/MSP; input from the webcams (using e.g. motion analysis) allowed us to detect the presence of passers-by, their distance, and specific actions they made (e.g. waving). This was translated in sound output, such as the activation of music samples, creating an interactive environment that responded to all people walking through the corridor (see Figure 4).

The speakers were deployed in corridors in two different schools, allowing in situ evaluations with students encountering our system. In a series of iterations, we tried different combinations of input, mapping and sound output, step-by-step increasing our understanding of the curiosity principles, and the best way to implement them in this context. We designed and fine-tuned the interaction scenarios, the way in which the prototypes responded to the actions of the passers-by, to the user group (students from 18-22 years old).

We only present the more formally evaluated interaction scenarios; however, these scenarios were based on various iterations and explorations.
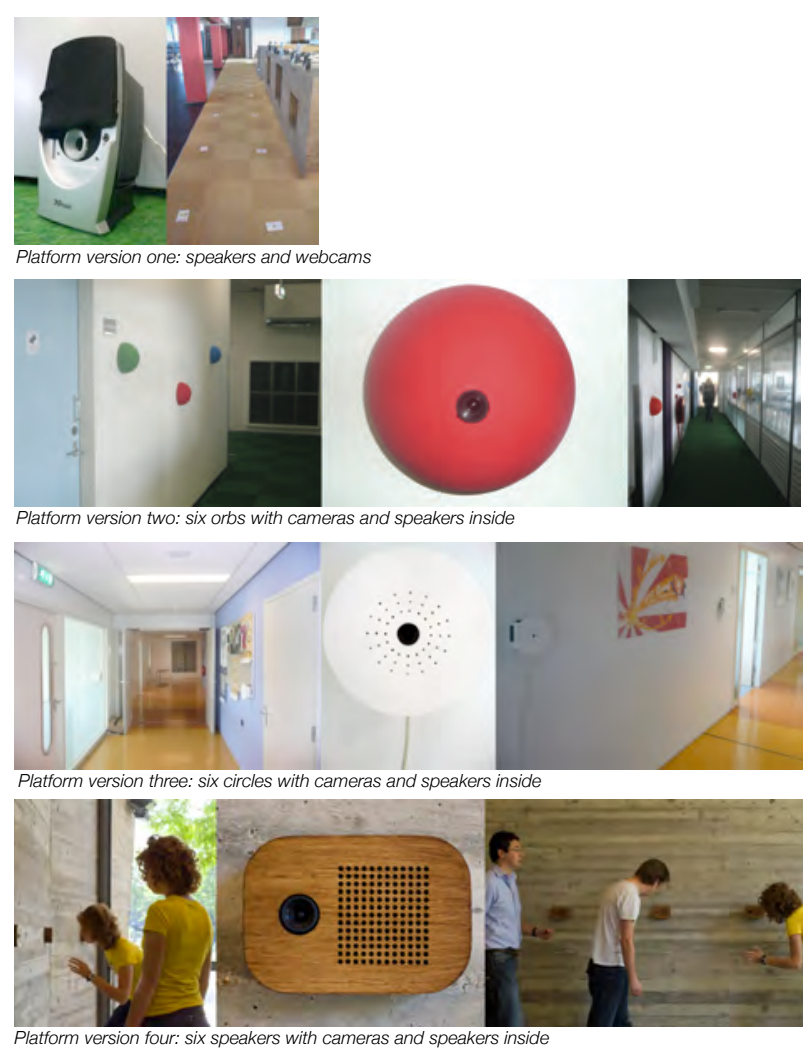

Figure 4: several versions of the speaker prototype; interactive loudspeakers that transformed action into sound.

\section{INTERACTION SCENARIOS}

Curiosity can be evoked through the following five principles, as described in the related work: novelty, partial exposure, complexity, uncertainty and conflict. Our goal was to explore if we could elicit curiosity and explorative behaviour through an interactive system, using those five principles.

We developed five interaction scenarios, mappings of the actions of passers-by, translated into sound output from the speakers. We expected each of those scenarios to influence the way students walked through the corridor. To trigger this behaviour, we used the five curiosity principles, with each scenario focusing on a specific principle. We now briefly present the implementations of the five interaction scenarios. They will be explained in more detail in the evaluation section. 


\section{Out-of-context animal sounds}

An 'out-of-context' situation was created, by playing the background noises from a farm. When a student would get close to a speaker, loud noises of scared animals would be given as feedback.

\section{Fragmented sounds}

We played fragmented audio samples from popular movies, in order to make passers-by curious for the 'total picture'. Audio fragments were played softly; inviting the students to listen closely and to 'remember' which movie it was taken from. After a few seconds of listening, the sample would fade out, and start from another speaker.

\section{Uncorrelated interaction through sound}

By coupling a variety of user actions to music samples in an uncorrelated way, we invited the users to make sense of the complex situation. We expected the users to explore different ways of interacting, by performing various actions and then listening to the results.

\section{Distorted sequences}

In this scenario we altered sequences, and thus distorted the expectations of the passers-by. Each speaker played a number; speaker one would say "One!", speaker two "Two", and so on. By disabling one of the last speakers, the expectation of the users was distorted: they would expect a "Six!", but the system would not respond at all.

\section{Cognitive distortion}

We created a conflicting situation by placing coloured footsteps on the floor in the corridor. Students passing a speaker would hear the colour they walked on, e.g. "Red!" while walking on the red footsteps. The last speaker always responded with the wrong colour, creating a mismatch between the real situation and the output from the system.

\subsection{Evaluation Setup}

We expected that the five interaction scenarios would result in a variety of user behaviours in the corridor: people would probably respond in different ways to the scenarios, walking in a different pattern than normally. In the following paragraphs, we describe the way in which we evaluated the influence of the interaction scenarios, and the behaviour they caused. The actual observed behaviour, and the most important conclusions, are covered in the subsequent sections.

The speaker platform was installed in a corridor in a vocational school. The corridor led to a self-study area; students walked through this corridor several times a day on their way to and from the self-study area.
The system was implemented in the school for five days (Monday-Friday). Due to the setup of the university's curriculum, different students were present each day: as a result of this, the majority of the students on a specific day had not encountered the system before.

Each day, a different interaction scenario was active: on Monday, the 'Animal-sounds' interaction, on Tuesday the 'Movie fragments', and so on. Each interaction was active for at least four hours; during these periods, the system worked standalone. On average, per day 25 students worked in the self-study area, passing the system 2-4 times each.

An observer was 'hidden' in an office at the end of the corridor; in addition, the cameras in the speakers recorded all movement in the corridor (visual only). The observer described all behaviour shown by passers-by; in addition, each passer-by was rated on a scale from 'no visible reaction to system, no change in walking pattern' to 'visible reaction to system, large change of walking pattern'. Special attention was given to the changes in behaviour: how the speakers influenced the natural behaviour of individuals and groups, and how groups of peers responded and interacted. The fact that a study was being conducted was not communicated to the students during the week.
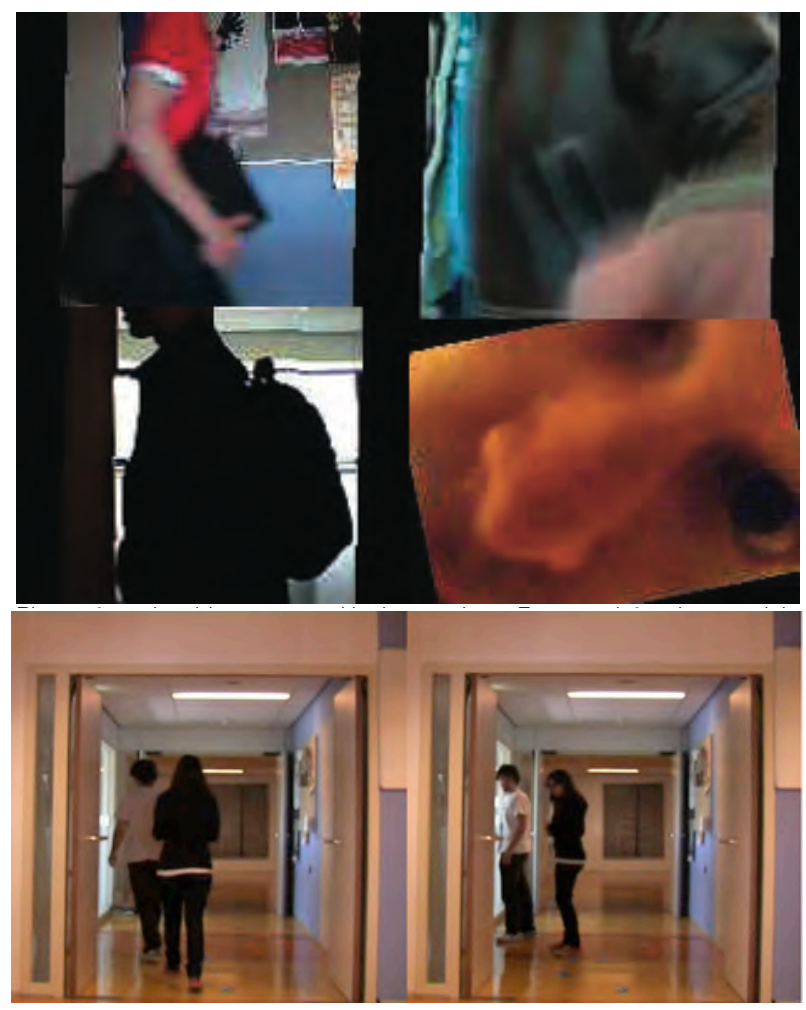

Figure 5: recordings of the user evaluation from the speaker prototypes, and from the observer's office. 


\subsection{Out-of-context animal sounds}

"Hmm, what is that?"

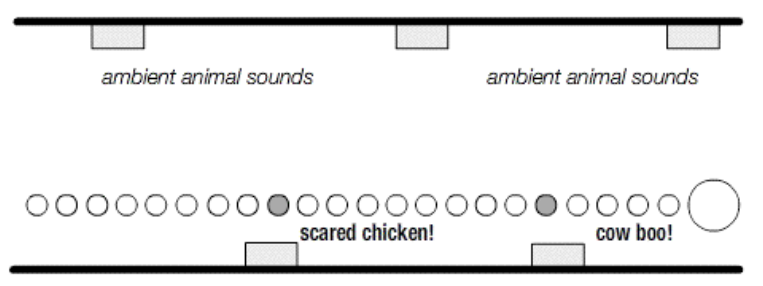

Figure 6: speakers produce background sounds related to a farm. If a student gets close to a speaker, the sound of a scared animal is played.

By creating a situation that is clearly out-of-context, we evoke curiosity through novelty. This means that something is new, or out of place, in a context and someone wants to know or feel 'what it is'. Novelty is associated with sensorial, novel and attention drawing experiences. Often, the curiosity evoked by novelty is quickly 'fulfilled', and then the curiosity is gone: the novelty has worn off.

Our assumption was that the interactive speakers would elicit short explorative behaviour. We expected students to stop and investigate the speakers, quickly understanding that a nearby movement would result in the sound of a scared animal. This shallowness would lead to a short spur of curiosity and exploration, before the student would be satisfied and walk on. Due to the sounds' obtrusiveness, we expected $\sim 75 \%$ of the students to stop and explore the system on their first encounter.

\section{Evaluation}

Example of a typical user encounter as observed during the evaluation:

Julia and Peter walk through the school to their next lesson. Julia mentions "Look, what are those?" She walks closer to a speaker, and suddenly a disturbed cow boos. Laughing, Peter walks to another speaker, and discovers a scared chicken. Together, they walk onward, scaring animals while they walk by.

Most of the students ( 70\%) stopped to explore the system on the first encounter: they briefly activated several speakers, hearing different animal sounds. After this short exploration they walked onward. On a next encounter most people walked by ignoring the system.

As expected, novelty works as a strong, but short, evoker of curiosity. The interactive speakers were clearly novel, drawing a lot of attention, and herewith eliciting explorative behaviour. After this novelty had been 'fulfilled', the speakers lost their strong attraction.
Novelty seems well suited to draw initial attention to an interactive system; however, this novelty quickly wears off, and additional principles have to be used if sustained or repetitive curiosity is needed.

\subsection{Fragmented sounds}

"I want to know what is hidden behind..."

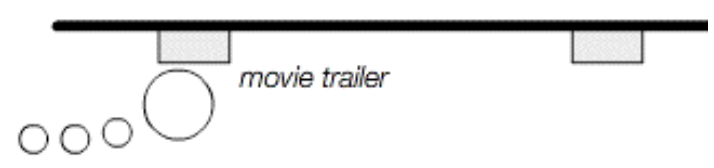

Figure 7: sound fragments from popular movies are softly played from a speaker. If a student stops to listen, the sound fades out and is played from an adjacent speaker.

By hiding or exposing certain parts of 'information', we make people curious for the 'total image'. In a way, we point out a gap in someone's knowledge, we provide incomplete information, and if the user is interested in this information, he/she will want to fill this gap. This interest is important, and the 'size' of the gap: there should neither be too much exposed information, nor too little

We assumed that the partly exposed information would draw the attention of the students, since popular movie fragments were used. We expected them to stop, listen to the samples, and to recognize the origin after a few moments. Since this was a rather soft and unobtrusive interaction scenario, we expected $\sim 50 \%$ of the passers-by to stop and listen.

\section{Evaluation}

Example of a typical observed situation:

Sandra walks to her next lesson. On turning the corridor, she hears sound coming from an object attached to the wall. While walking, she eyes the objects without altering her stride, and walks onward.

Some passers-by $(\sim 10 \%)$ indeed stopped to listen to the sound samples; however, most of them walked on without stopping, just glancing at the speaker.

The system barely drew attention compared to the previous scenario; in addition, most of the students who did notice it just walked on. Apparently, the movie samples and the system did not evoke enough curiosity to elicit them to stop and explore. We assume that two factors have caused this; first of all, the passiveness of the system created a lot 
less novelty than the loud animal sounds in the first scenario. This system was less 'out-of-context', there was less contrast, and it drew less attention. In addition, the importance of the content became clear: most students did not seem to be interested or intrigued by the exposed information, thus hardly any curiosity was evoked. We learned that the information, and the hidden part, must really 'trigger': the passer-by should be interested in the information.

\subsection{Uncorrelated interaction through sounds}

\section{"How does that work?"}

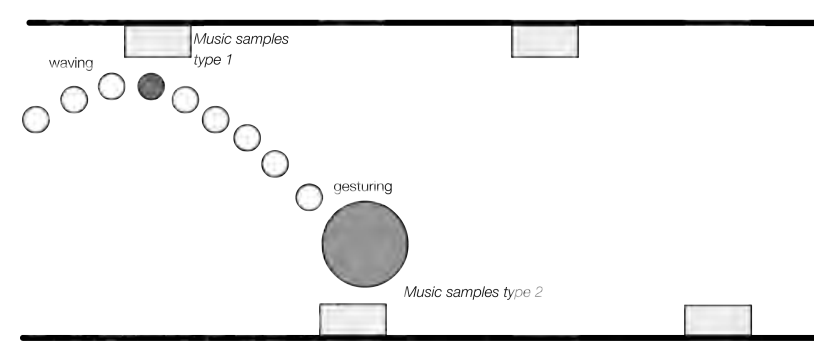

Figure 8: different actions in front of a speaker result in different sound samples. The correlation between a specific sound sample and action is seemingly random.

This scenario invited users to 'make sense of the situation', to explore and discover how the system worked. It was intended to be 'deeper' than the animal sound scenario, where users could only discover that movement resulted in scared animals. Here, every action resulted in a different sample, and even similar actions seemed to give ambiguous results.

We expected this scenario to elicit 'interpretive' behaviour: students trying all sort of actions, in order to discover what they could do with the system. Exploration, we assumed, would be more intense and take longer than the shallow exploration in the animal sound scenario. Based on previous explorations with music, we predicted that $\sim 60 \%$ of the passers-by would start exploring.

\section{Evaluation}

George and Eva walk through the corridor, being surprised by the sudden mixture of sound samples. George walks to a speaker, and waves in front of it, starting a new sample. Eva walks quickly to three speakers in a row, creating a symphony of different samples. After a minute of exploration and moving, the two walk onwards.

The high responsiveness of the system drew a lot of attention; many users ( $50 \%)$ started interacting with the system, discovering the link between action and music, and activating various speakers. Passers-by tried different sort of gestures, discussing with and mimicking each other in their explorations. On subsequent encounters, a true 'walk-through-and-use' phenomenon displayed itself: users would walk through the corridor, trying a few different gestures while passing each speaker.

The variable and ambiguous output elicited a lot of curiosity: passers-by tried to find out 'how the system worked', and while doing this they discovered additional ways of interacting (e.g. waving, walking by, etc). Their discovery was rewarding (musical output), and at the same time they encountered a new aspect that had to be interpreted (new sample). This combination is important; if the discovery is tiny and boring, then the reward is not worth the exploration, and it will not trigger new cycles of exploration and discovery.

\subsection{Distorted sequences}

"Hey, wasn't it supposed to...?"

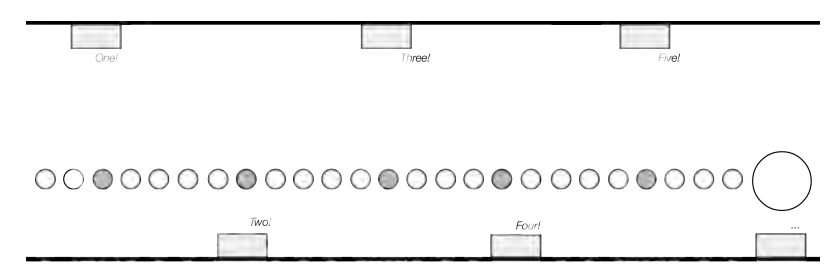

Figure 9: walking through the corridor results in a sequence of numbers - "One!" from the first speaker,

"Two!" from the second, and so on. One of the last speakers is quiet, not responding to the passer-by at all.

By altering the sequence, and thus distorting the expectations, we created a mismatch between expectation and perceived situation. Previous design explorations had shown us that passers-by immediately created expectations while walking through a corridor; not fulfilling these expectations did often create doubt and surprise.

We assumed that the first speaker would elicit a short span of novelty: a speaker playing "One" when you walk by is clearly out-of-context. Since this was all the speaker did, we expected students to walk on after a few seconds of exploration. We predicted that most students would notice the 'quiet' speaker, commenting on it to peers and perhaps even stopping to try and activate it. We expected $\sim 30 \%$ of the students to interact with the system.

\section{Evaluation}

Maria and Martin walk through the corridor, creating a 'one-two-three' pattern. After a brief exploration, they realise that movement results in a number, and they walk on - 'four-five-..[silence]..'. Slightly roused, Martin shouts 'six, you idiot!'. Maria comments 'it must be broken'. On a next encounter, Martin softly mimics the voices 'One, two, three' while walking by. Speaker four is quiet, 
and Martin stops to wave in front of the speaker. After a few seconds, he walks on, again stopping at the (now active) 'Six', looking surprised.

The results of this evaluation were unexpected: multiple passers-by started counting aloud with the system, even on first encounters, and were visually and verbally surprised by the lack of reaction from the silent speakers. In general, many users ( 20\%) walked back and started to wave in front of the speaker. Some users even started to talk to the speaker and peers, usually ending with an 'it must be broken'.

The counting numbers pattern, and the expectations it immediately created, proved to be powerful for creating uncertainty and curiosity: users were clearly distressed by the mismatch with their expectations, walking back and trying to activate the speaker. From their comments, it seemed that they did not know if their expectations were wrong, or if the system was wrong.

\subsection{Cognitive distortion}

"No, that's wrong!"

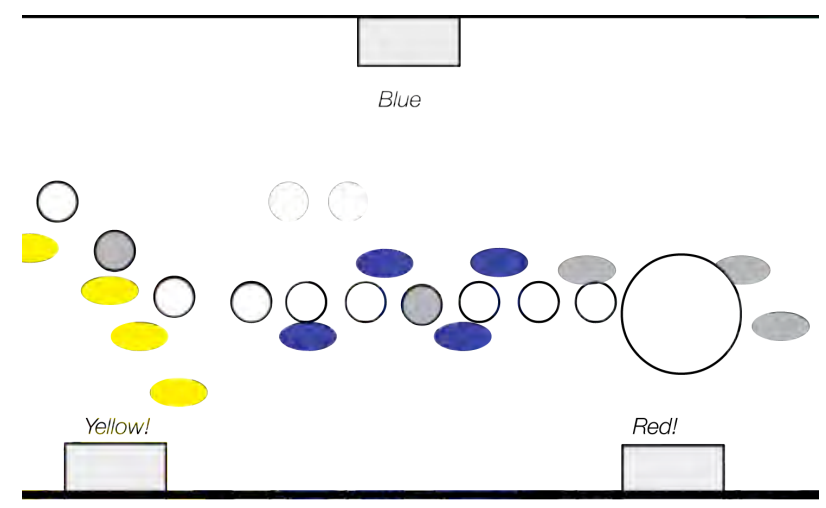

Figure 10: footsteps create coloured areas in the corridor. When a student passed a speaker, the corresponding colour would be played. The last speaker announced an incorrect colour.

This interaction scenario revolves around conflict: conflicting experiences and incompatibility between ideas. It seems similar to the distorted sequences scenario; however, where the former is about surprise and the doubt of what is going on, this scenario clearly presents something that is 'not right'. The paintings of Escher are a perfect example of curiosity through conflict: things should not be possible, yet they seem possible, and one wonders why.

We assumed that this scenario, due to its relative obtrusiveness, would again create a short span of novelty, though quickly fulfilled. In addition, we predicted that this scenario would evoke a short 'realisation' of conflict for $\sim 30 \%$ of the passers-by: a surprised look from the students, before they would continue their walk.

\section{Evaluation}

Liss and Catherine walk through the corridor, following the red footsteps. The speakers respond with "Red! Red! Red! Blue!". Liss walks on without noticing; Catherine looks back with a pondering expression on her face.

In this evaluation, we observed three types of behaviour: Most of the people ( 60\%) almost ignored the system, just looking at the speakers while crossing the corridor. Some of the others ( 20\%) commented on the system: "It's somehow detecting on which colours we walk - but it isn't correct all the time". The remainder of the passersby started to explore how the system worked: they tried different ways of 'walking-by', in order to analyse how the system measured things. However, a large part of the passers-by ( $50 \%$ ) focused their attention on the coloured footsteps, jumping from colour to colour or just following the path of footsteps.

This interaction scenario was not efficient; it did attract some attention, but it hardly elicited explorative behaviour. We assume that the coloured footsteps were the main reason for this: people focused a lot of their attention on those steps, adapting their walking pattern to the path of the footsteps. This seemed to result in less attention for the speakers and the sound output.

While setting up the system, an interesting observation was made: the footsteps were not yet on the floor, but the system did already respond to passers-by. Students assumed that the speakers responded to the colour of their clothes, and commented that the system was either smart (when correct), or stupid (when wrong).

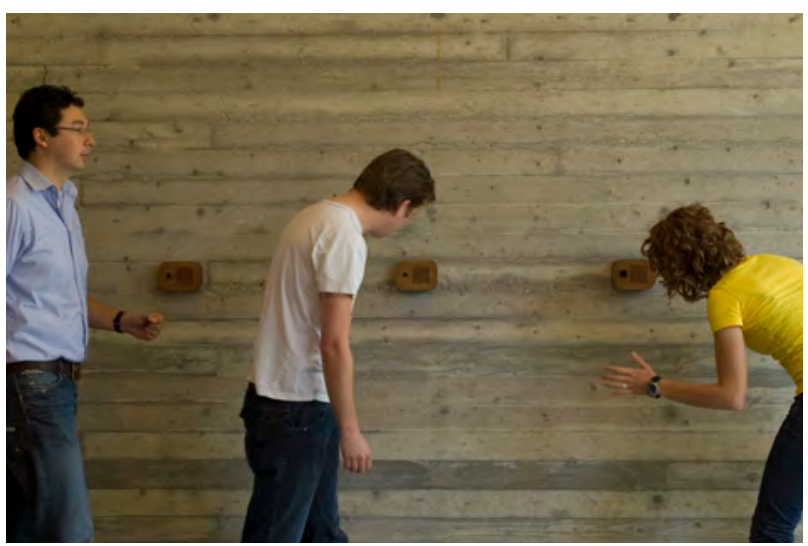

Figure 11: composition of different actions as observed during the user evaluation 
Table 1: curiosity principles and interaction scenarios, and the relation between them. Big crosses represent a strong relation, small crosses a weak relation.

\begin{tabular}{|l|l|l|l|l|l|}
\hline & Novelty & Partial Exposure & Complexity & Uncertainty & Conflict \\
\hline Animal sounds & $X$ & & & & \\
\hline Fragmented sounds & $x$ & $X$ & & & \\
\hline Uncorrelated interaction & $x$ & & $X$ & & \\
\hline Distorted sequence & $x$ & & $x$ & $X$ & $x$ \\
\hline Cognitive dissonance & $x$ & & $x$ & $x$ & $X$ \\
\hline
\end{tabular}

\subsection{Conclusion}

Overall, the interactive system elicited curiosity and explorative behaviour; clear differences could be observed in the amount and sort of behaviour, depending on the active interaction scenario.

Each of the interaction scenarios revolved around a specific principle; naturally, overlaps did occur. Table 1 gives an indication of those overlaps.

We have presented five interaction scenarios, that all elicited curiosity and explorative behaviour in different ways and with different levels of success. For our goal, target group and context, three curiosity principles were powerful: novelty, complexity and uncertainty. Other principles would probably be suited for other target groups and contexts. In addition, the type of explorative behaviour that one wants to elicit influences the choice of curiosity principles.

Based on the evaluations, we conclude that it is possible to elicit curiosity using 'isolated' curiosity principles. However, combining principles in order to catch and hold the attention of the passer-by is more powerful. For example, one could create a clear out-of-context situation (novelty) to draw the attention of the user, then 'drag' the user into cycles of exploration and discovery (complexity). On a next visit, the system could respond in a different way, creating doubt (uncertainty) and starting a new cycle of exploration and discovery (complexity).

\section{INFLUENCE FACTORS}

In our design iterations, we identified other factors that strongly influenced if and how curiosity was evoked, and the sort of behaviour it elicited. We describe them in the following section.

\subsection{User}

The user, the specific person that encounters an interactive system, naturally influences the encounter and the behaviour. This user is the one who has to make the decision to interact or to walk on, and decides how to interact with the system.

We observed three main factors:

- user traits and characteristics: some people are simply more curious and open to new experiences than others; some are shy, others proudly try out everything they see, etc.

- curiosity openness: if a person is running to catch a train, or talking on the telephone, then the chance that this person becomes curious and starts to explore is very small.

- expectation: the expectation of the person influences his/her actions as well. If the person knows that there is a speaker responding to movement, he/she will act differently compared to an ignorant user.

\subsection{Context}

The context, both the location and the current status, influence the elicited curiosity for a system. For example, a crowded train station affords totally different behaviour than a quiet alley.

We identified two factors:

physical characteristics: the location, and the physical characteristics of this location, influence the type of passers-by, the way they walk, their mindset, and so on. A school is different than a shopping mall, and a corridor in a school is different than a staircase.

social environment and conditions: the current social circumstances influence the way people react to the system. In our school, if a group of people was watching the corridor, passers-by behaved totally differently than in the early, quiet mornings.

\subsection{Memory}

It is important to realise that curiosity, and the exploration with the system, always creates a memory. If this memory is negative (e.g. the user is highly confused or annoyed by the system), then this will influence the behaviour on the next encounter. 


\section{DISCUSSION}

In the last years, playfulness, attraction and enjoyable experience design are being studied from a variety of perspectives. Korhonen et al. (2009) for example provide an overview of many playful principles. We have picked apart one of these principles, curiosity, and examined how it can be incorporated in design.

The implementation covered in this article provided us with insights and an increased understanding of eliciting curiosity - but it was only one case study. More case studies are required to explore and study curiosity and related experiential factors in depth.

Our set of design explorations has resulted in an initial model, that needs to be developed further in future design research studies. In our current work, we are exploring the validity of our insights in other applications, and are implementing other principles in order to motivate playful experiences.

The strength of this work is that the designs were tested in the field, without the passers-by knowing that they were participants: this ignorance is mandatory when studying curiosity in first encounters. The importance of this ignorance became clear in initial explorations, when we interviewed passers-by and recorded them with a hidden camera: as soon as the students realised that an evaluation was going on, they told their peers, and the explorative atmosphere changed radically. Students started to make fun of each other and the system, acting stupidly or overly curious. In a different school, we did not give any clues that an evaluation was being executed. Here, students told some of their peers about this odd system as well, creating foreknowledge for the passers-by. However, this caused a 'common exploration' to start: passers-by started discussing what they thought about the system, what they had heard from others, and so on. This sort of group exploration, and the change in group dynamics, was exactly what we wanted to study; interviewing during the study or otherwise indicating that an evaluation was being performed would have prevented this.

While not covered in this paper, we have also observed the behaviour of students on subsequent encounters with the system: how did they behave when they encountered the system again, and how did the group dynamics change over time? Design explorations to stimulate repetitive curiosity are currently being conducted, incorporating mechanisms from education and gaming such as scaffolding.

It is important to mention that we are aware of the analytical choices that we have made: curiosity in play is never isolated or totally independent, it is always interconnected with enjoyment, challenge, and other principles. However, to start with studying curiosity in depth, we decided to strip all other principles from our interaction designs, attempting to design only for curiosity. Curiosity is a huge, intertwined concept, but one has to start somewhere. We decided to start with this isolated, relatively small curiosity, and we will expand from here. Eventually, we will try to broaden our understanding of, and the way in which we study curiosity to full experiential experiences.

\section{CONCLUSION}

We have presented an explorative study on how to design for curiosity through interactive systems. A literature survey resulted in a list of curiosity principles. Iterations of design explorations and evaluations have provided insights in how such principles can be implemented in design. We have given examples and reflect upon evaluations of those principles in a specific context.

All principles have a powerful influence on people's behaviour, depending on the specific application and context. Combining these principles, and finetuning them for a specific target group, can lead to even more powerful results. The combination of novelty, complexity and uncertainty for example creates a promising, repeating stimulant for exploration.

In future work we will examine how to apply these principles in different ways, in order to seduce school youth to change their behaviour in a playful manner.

\section{REFERENCES}

Bekker, T., Sturm, J. and Eggen, J. (2010). Designing Playful Interactions for social interaction and physical play, Personal and Ubiquitous Computing, 14(5), 285-296

Berlyne, D.E. (1960). Conflict, Arousal and Curiosity, McGraw-Hill, New York

Berlyne, D. E. (1967). Arousal and reinforcement, D. Levine (Ed.), Nebraska symposium on motivation, Lincoln: University of Nebraska Press.

Berlyne, D. E. (1971). Aesthetics and psychobiology. New York: Appleton-Century-Crofts. Cialdini, R.B. (2001). Influence: science and practice, Allyn \& Bacon, London, 2001

Garris, R., Ahlers, R. and Driskell, J.E. (2002). Games, motivation, and learning: $A$ research and practice model, Journal of Simulation \& Gaming, Vol. 33, No. 4, Sage Publications, 2002, pp. 441467

Gaver, W.W., Beaver, J. and Benford, S. (2003). Ambiguity as a resource for design, Proceedings of CHI 2003, Florida, USA, 2003, pp. 233-240 
Harter, S. (1981). A new self-report scale of intrinsic versus extrinsic orientation in the classroom: Motivational and informational components. Developmental Psychology, 17, 300312.

Kashdan, T.B., Rose, P. and Fincham, F.D. (2004). Curiosity and Exploration: Facilitating Positive Subjective Experiences and Personal Growth Opportunities, Journal of Personality Assessment, vol. 82 iss. 3, Lawrence Erlbaum Associates, 2004, pp. 291-305

Korhonen, H., Montola M., and Arrasvuori J. (2009). Understanding Playful Experiences Through Digital Games, Proc. of DPPI'09, Compiegne, France, pp.274-285.

Loewenstein, G. (1994). The psychology of curiosity: a review and reinterpretation, Psychological Bulletin, vol. 116, 1994, pp. 75-98

Rauterberg, M. (1995). About a Framework for information and information processing of learning systems, Proceedings of IFIP conference on Information System Concepts, London, UK, 1995, pp. 54-69
Romero, N. Sturm, J., Bekker, T.,. de Valk, L. and S. Kruitwagen. (2010). Playful persuasion to support older adults' social and physical activities. Interact. Comput. 22, 6 (November 2010), 485-495. TheFunTheory (2009). Piano Stairs, http://www.thefuntheory.com (November 2, 2010).

Vorst, R. v.d. (2007). Nieuwsgierigheid: hoe wij elke dag worden verleid (in dutch), Nieuw Amsterdam, Netherlands, 2007

Yee, N. (2007). Motivations of Play in Online Games, Journal of CyberPsychology and Behaviour, Vol. 9, 2007, pp. 772-775

Zheng, S., Bromage, A., Adam, M., Scrivener, S.A.R. (2007). Suprising Creativity: A Cognitive Framework for Interactive Exhibits Designed for Children, in Proceedings of C\&C'07, Washington, USA, 2007. 